\title{
Impact of Skull Defects on the Role of CTA for Brain Death Confirmation
}

\author{
(DD.M. Nunes, (D) A.C.M. Maia Jr, (D)R.C. Boni, and (D)A.J. da Rocha
}

\begin{abstract}
BACKGROUND AND PURPOSE: Intracranial pressure modifications caused by a skull defect, such as craniectomy or craniotomy, may change the hemodynamics and decrease the accuracy of CTA to confirm brain death. This study aimed to evaluate the impact of a skull defect and the interpretation criteria of images on this diagnostic test.
\end{abstract}

MATERIALS AND METHODS: A series of consecutive patients with a clinical diagnosis of brain death underwent CTA (case group), while the control group comprised patients with acute ischemic stroke in the same period. CTA criteria adopted to confirm brain death were the absence of opacification of the M4 branches and internal cerebral veins. The evaluation also included the presence of "stasis filling." Cases were stratified as intact skull, craniotomy, and craniectomy. Three neuroradiologists evaluated all examinations independently.

RESULTS: In the case group, according to the Frampas criteria, the sensitivity of CTA to confirm brain death was $95.5 \%$ in patients with intact skull, $87.5 \%$ with craniotomy, and $60 \%$ with craniectomy. False-negative diagnoses of brain death were $15.6 \%$, related to stasis filling in $71.4 \%(P<.001)$. However, according to the "modified Frampas criteria," the sensitivity of CTA to confirm brain death was $100 \%$ in patients with intact skull, $93.8 \%$ with craniotomy, and $80 \%$ with craniectomy. False-negative diagnoses of brain death were found in $6.2 \%$ of patients, and there was no stasis filling. CTA showed $100 \%$ specificity in the control group. There were no disagreements among observers.

CONCLUSIONS: CTA had a high diagnostic accuracy and reproducibility to confirm brain death in patients with an intact skull. The modified Frampas criteria increased the sensitivity of CTA, particularly in patients with a skull defect. A concurrent skull defect, especially craniectomy, can decrease the sensitivity of CTA to confirm brain death.

ABBREVIATIONS: $B D=$ brain death; $I C M=$ iodinated contrast medium; $I C V=$ internal cerebral vein; $S D=$ skull defect; $S F=$ stasis filling

B rain death $(\mathrm{BD})$ is the result of diffuse, progressive, and irreversible brain damage clinically characterized by a deep unresponsive coma and the absence of spontaneous ventilation and brain stem reflexes. ${ }^{1-3}$ Although the clinical criteria for the diagnosis of brain death assess the absence of brain stem functions, some countries have used the concept of global brain death, adding ancillary tests to show the absence of brain circulation or absence of electric activity or absence of metabolic activity, including the cerebral hemispheres. ${ }^{4-6}$ Circulatory evaluation is commonly used because it is subject to less pharmacologic inter-

Received March 2, 2019; accepted after revision May 13.

From the Section of Neuroradiology (D.M.N., A.C.M.M.Jr., A.J.d.R.) and Section of Organ Procurement Organization (R.C.B.), Santa Casa de Misericórdia de São Paulo, São Paulo, SP, Brazil.

Please address correspondence to Douglas Mendes Nunes, MD, Santa Casa de Misericórdia de São Paulo, Serviço de Diagnóstico por Imagem, Rua Dr. Cesário Motta Junior 112, Vila Buarque, São Paulo, SP, Brazil, 01221-020; e-mail:

douglasmendesnunes@gmail.com

http://dx.doi.org/10.3174/ajnr.A6100 ference (eg, the use of sedatives and occurrence of severe electrolyte and metabolic disorders). ${ }^{7,8}$

Conventional angiography is the criterion standard to evaluate the circulatory system..$^{9-11}$ However, because this method is invasive, is not widely available, and requires specialized neuroradiologic expertise to perform and interpret the results, other alternatives have been investigated, including CTA. The accuracy of CTA for a BD diagnosis was validated by Frampas et $\mathrm{al}^{12}$ through the absence of intracranial arterial opacification of the distal branches (M4) and internal cerebral vein (ICV), both evaluated in the venous series of CTA, showing moderate sensitivity $(85.7 \%)$ and high specificity (100\%). Other studies have confirmed similar accuracies. ${ }^{13-18}$ Nevertheless, the interpretation criteria of the images used by Frampas et $\mathrm{al}^{12}$ limited the applicability of CTA to confirm BD due to patients with false-negative findings (14.3\%).

According to the Monro-Kellie doctrine, intracranial pressure is the result of the balance among the CSF, blood, and brain parenchyma inside the skull, a rigid compartment. Increased intra- 
cranial pressure results in pressure compensation through the displacement of the CSF to the spinal subarachnoid space, followed by intracranial vascular collapse. This collapse occurs initially in the venous compartment because of the increased compliance and lower intraluminal pressure and then in the arterial compartment, with consequent brain damage. ${ }^{19-21}$

Therefore, a skull defect (SD) in patients with suspected $\mathrm{BD}$, such as extensive fracture or operation (craniectomy or craniotomy), may decrease the intracranial pressure, changing the intracranial hemodynamics and decreasing the diagnostic accuracy of ancillary tests that evaluate brain circulation. ${ }^{10,22,23}$ However, there are no specific studies evaluating the accuracy of CTA for BD confirmation in patients with SD, to our knowledge.

Our aim was to evaluate the impact of SDs and the interpretation criteria of imaging on the accuracy of CTA for BD confirmation.

\section{MATERIALS AND METHODS Study Design}

This cross-sectional and observational study was conducted at a single hospital center (Santa Casa de Misericordia de São Paulo) between June 2012 and July 2014. A case series of consecutive patients with a clinical diagnosis of $\mathrm{BD}$ (reference standard determination of BD) was evaluated according to the legal requirement, ${ }^{24}$ the Brazilian BD protocol, which consists of 2 clinical examinations and some confirmatory ancillary tests, namely, transcranial Doppler (47 patients), conventional angiography (2 patients), and nuclear medicine (1 patient). In addition to the Brazilian BD protocol, these patients underwent CTA, the results of which did not interfere with the final diagnosis regarding $\mathrm{BD}$. Due to the similarity between the stroke and BD protocols, the control group consisted of 22 patients with ischemic stroke who were candidates for thrombolysis and underwent CTA before any therapeutic proposal, in the same period. The institutional review board approved this study.

All patients older than 2 years of age with a $\mathrm{BD}$ clinical diagnosis, evaluated according to the legal requirements ${ }^{24}$ of the Brazilian BD protocol, were enrolled after their legal guardian and 2 witnesses agreed to participate in the study by signing the free and informed consent form. Patients were excluded when they had a mean arterial pressure lower than $80 \mathrm{~mm} \mathrm{Hg}$, if the guardians did not authorize study enrollment, if contraindications or the inability to receive intravenous iodinated contrast medium (ICM) was present, or if the technical quality of the images was deemed inadequate. Demographic and clinical data, including age, sex, cause of $\mathrm{BD}$, and the time interval between the $\mathrm{BD}$ diagnosis and CTA, were collected in a data base.

\section{Technical Data}

All examinations were acquired in a 64-detector row CT scanner (Version v2.6.2.22004; Brilliance ${ }^{\mathrm{TM}}$ CT 64-channel scanner; Philips Healthcare, Best, the Netherlands) with a minimum dose of ionizing radiation ${ }^{25}$ following the CTA protocol proposed by Frampas et al. ${ }^{12}$

After lateral topography, 3 similar acquisitions were planned starting at the $\mathrm{C} 1-\mathrm{C} 2$ level to the cranial vertex. The first acquisition was an NCCT scan $(120 \mathrm{kV} ; 350 \mathrm{~mA})$. Iso-osmolar, nonionic intravenous ICM (iopromide, Ultravist 300; Bayer HealthCare, Berlin, Germany) was injected through the right antecubital vein at a rate of 4-6 mL/s using a dual-head power injector (Medrad Stellant ${ }^{\circledR}$ D, Bayer HealthCare, Indianola, Pennsylvania) and a 16- to 22-ga catheter, with a pressure limit of 325 psi, depending on the age range. The total volume administered throughout the protocol was calculated on the basis of the estimated weight of the patient $(2.0 \mathrm{~mL} / \mathrm{kg}$, reaching a maximum of $50 \mathrm{~mL})$. The second (arterial) and third (venous) acquisitions $(120 \mathrm{kV}, 185 \mathrm{mAs}$, $0.67-\mathrm{cm}$ thickness, $0.33-\mathrm{cm}$ increments, FOV of $450 \mathrm{~mm}$, and automatic settings for pitch and release) were performed, starting 20 and 60 seconds after the ICM injection commenced.

\section{Image Analysis}

To evaluate hemodynamic repercussions resulting from the SD, we divided the patients into 2 different groups: the intact skull and SD groups. The patients with SDs were divided into 2 subgroups, those with craniotomy and craniectomy.

The NCCT series confirmed the adequate passage of intravascular ICM in all patients by identifying opacification of the superficial temporal arteries and upper orbital veins during the examination as well as differentiating between spontaneously hyperattenuating vascular content and delayed, weak, persistent, and progressive vascular opacification (pseudo-subarachnoid hemorrhage versus true vascular opacification).

The arterial and venous series enabled the assessment of vessel opacification in the intracranial arterial and venous compartments via CTA based on MIP postprocessing algorithms (thickness: $10 \mathrm{~mm}$ ) in workstation (Extended Brilliance ${ }^{\mathrm{TM}}$ Workspace, Version 3.5.0.2250; Philips Healthcare).

The progressive opacification of the intracranial arterial compartment between early (arterial) and delayed (venous) acquisitions of CTA was analyzed. The delayed, weak, persistent, and progressive opacification of the intracranial arterial compartment found between early (arterial) and delayed (venous) acquisitions of CTA is known as "stasis filling" (SF). This phenomenon is commonly observed in angiographic studies. ${ }^{10,26,27}$

Three neuroradiologists with experience in the interpretation of CTA studies $(9,22$, and 23 years, respectively) evaluated all the examinations independently using simultaneous viewing of images of the NCCT, arterial (early), and venous (delayed) CTA series. These findings were assessed to generate a single consensus report, which was compared with the BD clinical diagnosis (reference standard determination of $\mathrm{BD}$ ).

Two sets of criteria were used to confirm BD by CTA. The original criteria proposed by Frampas et al, ${ }^{12}$ which consist of the absence of opacification of the M4 branches and ICV, both evaluated in the venous series of the CTA; and another set, called in this study the "modified Frampas criteria," consisting of the absence of opacification of the M4 branches in the arterial phase and ICV in the venous phase of the CTA. The rationale for using these modified criteria is to avoid false-negative findings due to SF because opacification of the intracranial arterial compartment was evaluated in the arterial phase of CTA. 


\begin{tabular}{|c|c|c|c|c|c|}
\hline \multirow[b]{2}{*}{ Variable } & \multirow[b]{2}{*}{$\begin{array}{c}\text { Controls, ISk } \\
\quad(n=22)\end{array}$} & \multicolumn{3}{|c|}{ Patients with BD } & \multirow[b]{2}{*}{$P$ Value } \\
\hline & & $\begin{array}{c}\text { ISk } \\
(n=22)\end{array}$ & $\begin{array}{c}\text { Craniotomy } \\
(n=16)\end{array}$ & $\begin{array}{c}\text { Craniectomy } \\
(n=10)\end{array}$ & \\
\hline Age (yr) & $57.6 \pm 16.4$ & $42.8 \pm 21.9$ & $43.7 \pm 18.8$ & $47.4 \pm 9.2$ & .811 \\
\hline Male sex & $12(54.5)$ & $13(59.1)$ & $8(50.0)$ & $7(70.0)$ & .600 \\
\hline Etiology & & & & & .287 \\
\hline SAH & $0(0.0)$ & $6(27.3)$ & $8(50.0)$ & $3(30.0)$ & \\
\hline TBI & $0(0.0)$ & $4(18.2)$ & $4(25.0)$ & $5(50.0)$ & \\
\hline HS & $0(0.0)$ & $4(18.2)$ & $1(6.3)$ & $0(0.0)$ & \\
\hline IS & $22(100)$ & $4(18.2)$ & $0(0.0)$ & $0(0.0)$ & \\
\hline Tumor & $0(0.0)$ & $1(4.5)^{\prime}$ & $2(12.5)$ & $1(10.0)$ & \\
\hline GW & $0(0.0)$ & $1(4.5)$ & $1(6.3)$ & $0(0.0)$ & \\
\hline Other & $0(0.0)$ & $2(9.1)$ & $0(0.0)$ & $1(10.0)$ & \\
\hline Time interval from BD to CTA (min) & - & $\begin{array}{c}659 \\
(274-964)\end{array}$ & $\begin{array}{c}670 \\
(269-1013)\end{array}$ & $\begin{array}{c}483 \\
(236-713)\end{array}$ & .618 \\
\hline
\end{tabular}

Note:- SAH indicates subarachnoid hemorrhage; TBI, traumatic brain injury; HS, hemorrhagic stroke; IS, ischemic stroke; GW, gunshot wound; ISk, intact skull; BD, brain death; -, no data. ${ }^{a}$ Data are presented as No. (\%), except for age (mean \pm SD) and time (median and quartile). P values are for comparison of the subgroups (ISk, craniotomy, and craniectomy).

\section{Statistical Analysis}

Categoric variables were described by the absolute and relative frequencies and were compared using the $\chi^{2}$ test. The age and time interval between the clinical diagnosis of BD and CTA were assessed for normality using graphic methods and skewness and kurtosis values. Age was described using means and standard deviations and was compared using the Student $t$ test for independent samples. The time interval was described using medians and quartiles and was compared using the Mann-Whitney test.

The accuracy of CTA in each group according to the presence and type of SD was determined using contingency tables and by calculating the sensitivity, specificity, and positive and negative predictive values. Interobserver agreement was evaluated using the Cohen $\kappa$, with the following interpretation: $<0$, no agreement; $0-0.19$, very poor agreement; $0.20-0.39$, poor; $0.40-0.59$, moderate; $0.60-0.79$, high; and $0.80-1.00$, very high agreement.

All tests were 2 -tailed, and $P$ values $<.05$ were considered significant. Statistical analysis was performed using $\mathrm{R}$ statistical and computing software (http://www.r-project.org), SPSS (Version 24.0; IBM, Armonk, New York), and MedCalc for Windows (Version 15.2.0.0; MedCalc Software, Mariakerke, Belgium).

\section{RESULTS}

Seventy-two patients were evaluated, with 2 patients in the case group excluded because of poor image quality (poor contrast). Thus, 70 patients were included in the final analysis. The mean age of the sample was $48.3 \pm 18.9$ years (range, $2-84$ years), and $57.1 \%$ were men. Forty-eight patients in the case group were diagnosed with BD. By contrast, 22 patients in the control group were candidates for thrombolysis to treat ischemic stroke. The patients in the case group were comparatively younger $(44.0 \pm 18.6$ versus $57.6 \pm 16.4$ years; $P=.005)$, but no significant differences were found in sex between the groups ( $58.3 \%$ versus $54.5 \%$; $P=.766$ ).

The initial causes of coma were aneurysmal subarachnoid hemorrhage ( $n=17,35.4 \%)$, head trauma ( $n=13,27.1 \%)$, cerebral hemorrhage $(n=5,10.4 \%)$, brain tumor $(n=4,8.3 \%)$, ischemic stroke $(n=4,8.3 \%)$, and gunshot wound ( $n=2,4.2 \%)$. Three other cases $(6.3 \%)$ involved hydrocephalus due to ventriculoperitoneal shunt malfunction, hypoxic-ischemic encephalopathy after cardiorespiratory arrest due to respiratory causes, and a postoperative complication of Chiari malformation type I.
Twenty-six patients presented with SDs (16 craniotomies and 10 craniectomies). The median time interval between the final declaration of $\mathrm{BD}$ and $\mathrm{CTA}$ was 641 minutes (interquartile range, 269-883 minutes). Stratified analysis according to the presence and type of SD indicated no significant differences in age, sex, BD etiology, or the time interval between the declaration of $\mathrm{BD}$ and CTA. None of the patients in the control group had an SD (Table).

The 48 included patients in the case group were stratified according to the presence and type of SD. CTA demonstrated brain circulation arrest, defined according to the criteria of Frampas et $\mathrm{al}^{12}$ in 41 patients (41/48; sensitivity: $85.4 \%$ [95\% CI, $72.2-$ 93.9]). Among those with an intact skull, CTA was consistent with BD in 21 patients (21/22; sensitivity: 95.5\% [95\% CI, 77.2-99.9]). For those with an SD, CTA was consistent with BD in 20 patients (20/26; sensitivity: 76.9\% [95\% CI, 56.4-91.0]). The patients with an SD were subdivided into the craniotomy and craniectomy groups. CTA was consistent with BD in 14 patients with craniotomy (14/16; sensitivity: 87.5\% [95\% CI, 61.7-98.5]) and 6 patients with craniectomy (6/10; sensitivity: $60.0 \%$ [95\% CI, $26.2-$ $87.8]$ ). The categorization of patients with $\mathrm{BD}$ according to the criteria of Frampas et al, ${ }^{12}$ is summarized in Fig 1.

SF was diagnosed in 28 patients $(28 / 45,62.2 \%)$. Among these, 11 patients $(11 / 20,55.0 \%)$ had an intact skull, 10 patients $(10 / 15$, $66.7 \%)$ had undergone craniotomy, and 7 patients $(7 / 10,70.0 \%)$ had undergone craniectomy $(P=.390)$.

Seven patients $(7 / 48=14.6 \%)$ did not have a BD diagnosis from CTA using the criteria proposed by Frampas et al. ${ }^{12}$ Among these, 5 patients $(5 / 7,71.4 \%)$ presented with SF $(P<.001)$ and 2 patients $(2 / 7,28.6 \%)$ with craniectomy presented with arterial opacification of the distal branches (M4) in the arterial (early) acquisition of CTA. The false-negative CTA results were due to the SF phenomenon in 1 patient $(1 / 20,5.0 \%)$ with an intact skull, 2 patients $(2 / 15,13.3 \%)$ with craniotomy, and 2 patients $(2 / 10$, $20.0 \%)$ with craniectomy $(P=.208)$ (Fig 2).

However, when the so-called modified Frampas criteria were applied, CTA demonstrated brain circulatory arrest in 45 patients (45/48; sensitivity: 93.8\% [95\% CI, 82.8-98.7]). Among those with an intact skull, CTA was consistent with BD in 22 patients (22/22; sensitivity: 100.0\% [95\% CI, 84.6-100.0]). For those with an SD, CTA was consistent with BD in 23 patients (23/26; sensi- 


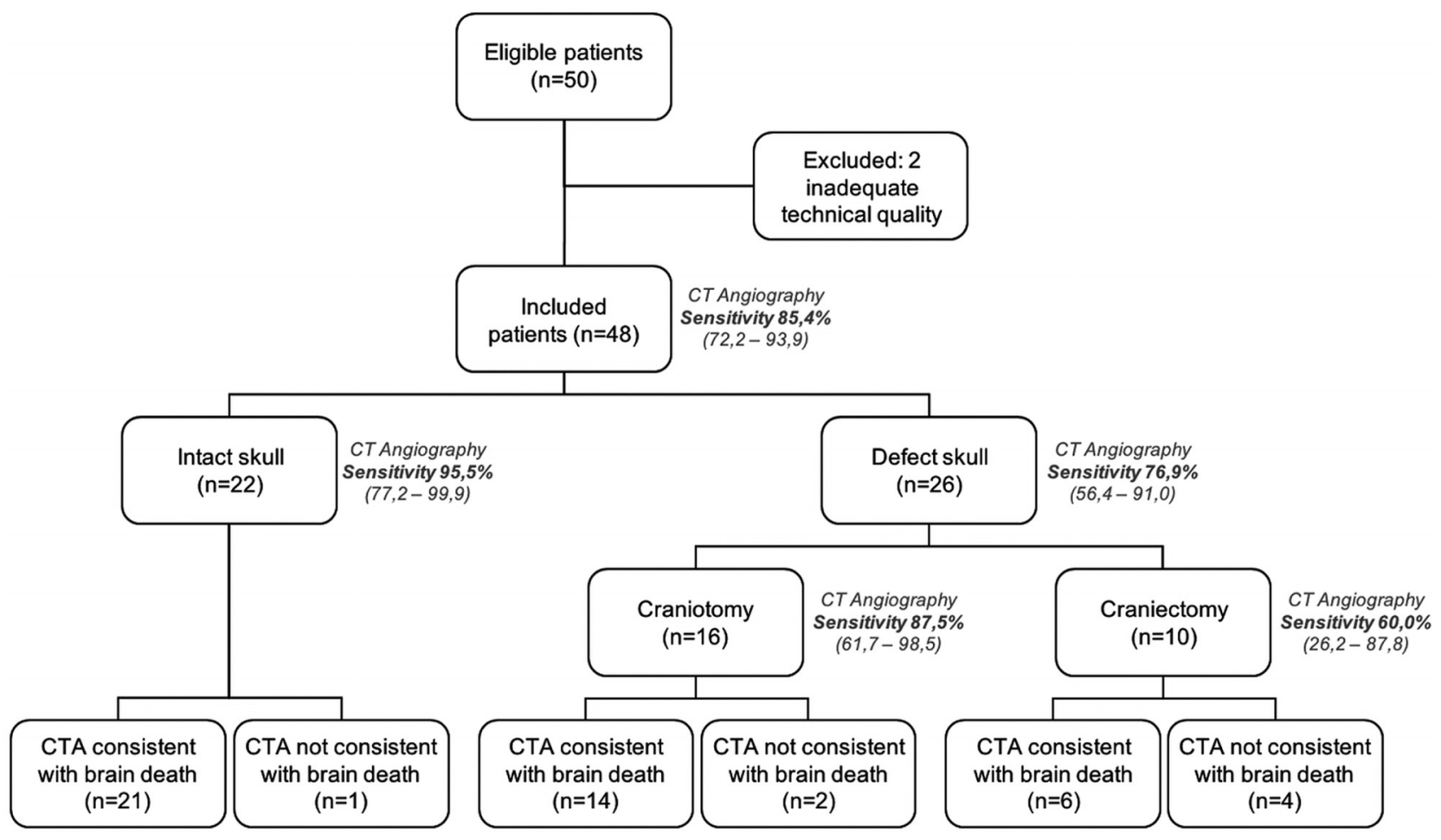

FIG 1. Flow chart of patients with a clinical diagnosis of brain death who underwent a CTA protocol proposed by Frampas et al, ${ }^{12}$ according to the presence and type of skull defect. Application of the interpretation criteria of the images proposed by Frampas et al.

tivity: $88.5 \%$ [95\% CI, 69.8-97.6]). The patients with an SD were subdivided into the craniotomy and craniectomy groups. CTA was consistent with BD in 15 patients with craniotomy $(15 / 16$; sensitivity: 93.8\% [95\% CI, 69.8-99.8]) and 8 with craniectomy (8/10; sensitivity: $80.0 \%$ [95\% CI, 44.4-97.5]). The categorization of patients with $\mathrm{BD}$ according to the modified Frampas criteria is summarized in Fig 3.

Even with these modified criteria, 3 patients $(3 / 48,6.2 \%)$ did not have a BD diagnosis using CTA. These patients with falsenegative findings of $\mathrm{BD}$ presented with arterial opacification of the distal branches (M4) in the arterial (early) acquisition of CTA: 1 patient $(1 / 3,33.3 \%)$ with craniotomy and 2 patients $(2 / 3$, $66.6 \%)$ with craniectomy.

There was a significant trend $(P=.037)$ toward higher falsenegative rates with more extensive SDs. Only 1 patient with craniectomy $(1 / 45,2.2 \%)$ showed opacification of the M4 branches and ICV in the arterial and venous series, respectively; in both, CTA criteria for BD were analyzed.

No association was found between the false-negative results and time interval between the diagnosis of $\mathrm{BD}$ and performance of CTA. The median time was 704 minutes (interquartile range 2471157 minutes) among the cases with an incorrect diagnosis by CTA and 641 minutes (interquartile range 270-857 minutes) among the cases with a correct diagnosis $(P=.405)$. Moreover, the percentage of incorrect diagnoses by CTA did not differ significantly among the time quartiles: first quartile, 93-270 minutes (2/12, 16.7\%); second quartile, 270-641 minutes (1/13, 7.7\%); third quartile, 641-857 minutes (1/11, 9.1\%); fourth quartile, 857-1577 minutes $(3 / 12,25.0 \%)(P=.551)$. These results indicated that the accuracy of CTA is not affected by the time between the diagnosis of $\mathrm{BD}$ and the performance of CTA.
The specificity of CTA to confirm BD was $100 \%$ in patients with an intact skull-that is, no false-positive cases were found. The specificity in patients with SDs could not be estimated because the control group included no patient with an SD. There were no disagreements in the CTA analysis among the evaluators, resulting in a perfect Cohen $\kappa$ of 1.0.

\section{DISCUSSION}

Despite the heterogeneity of protocols from CTA to confirm BD, the scientific literature has confirmed that CTA is a reliable, safe, fast, accessible, less invasive, reproducible, and auditable alternative ancillary confirmatory test in patients with the clinical criteria of BD. ${ }^{12-17,27-33}$ CTA has become increasingly common in the diagnosis of cerebral circulatory arrest and has already been licensed for use in many countries, such as Canada, Austria, Switzerland, France, the Netherlands, and Croatia. ${ }^{34}$

Frampas et $\mathrm{al}^{12}$ demonstrated the occurrence of cerebral circulatory arrest using CTA with moderate sensitivity (85.7\%) and high specificity (100\%), both by the absence of M4 branch and ICV opacification. Our study confirmed the reproducibility and diagnostic accuracy of CTA using the same criteria of 4 points proposed by Frampas et al, ${ }^{12}$ with a similar sensitivity (85.4\%), positive predictive value of $100 \%$, and absence of disagreement in the CTA analysis among the evaluators.

As Leclerc et $\mathrm{al}^{30}$ described and later studies have confirmed, ${ }^{13,31}$ the absence of ICV opacification constitutes the earliest and most sensitive (98.1\%) finding to confirm the diagnosis of BD with CTA. ${ }^{12}$ Our study also corroborated the high sensitivity $(97.98 \%)$ of the absence of ICV opacification. However, the exclusive evaluation of the intracranial venous compartment for the diagnostic documentation of BD with CTA should be made 


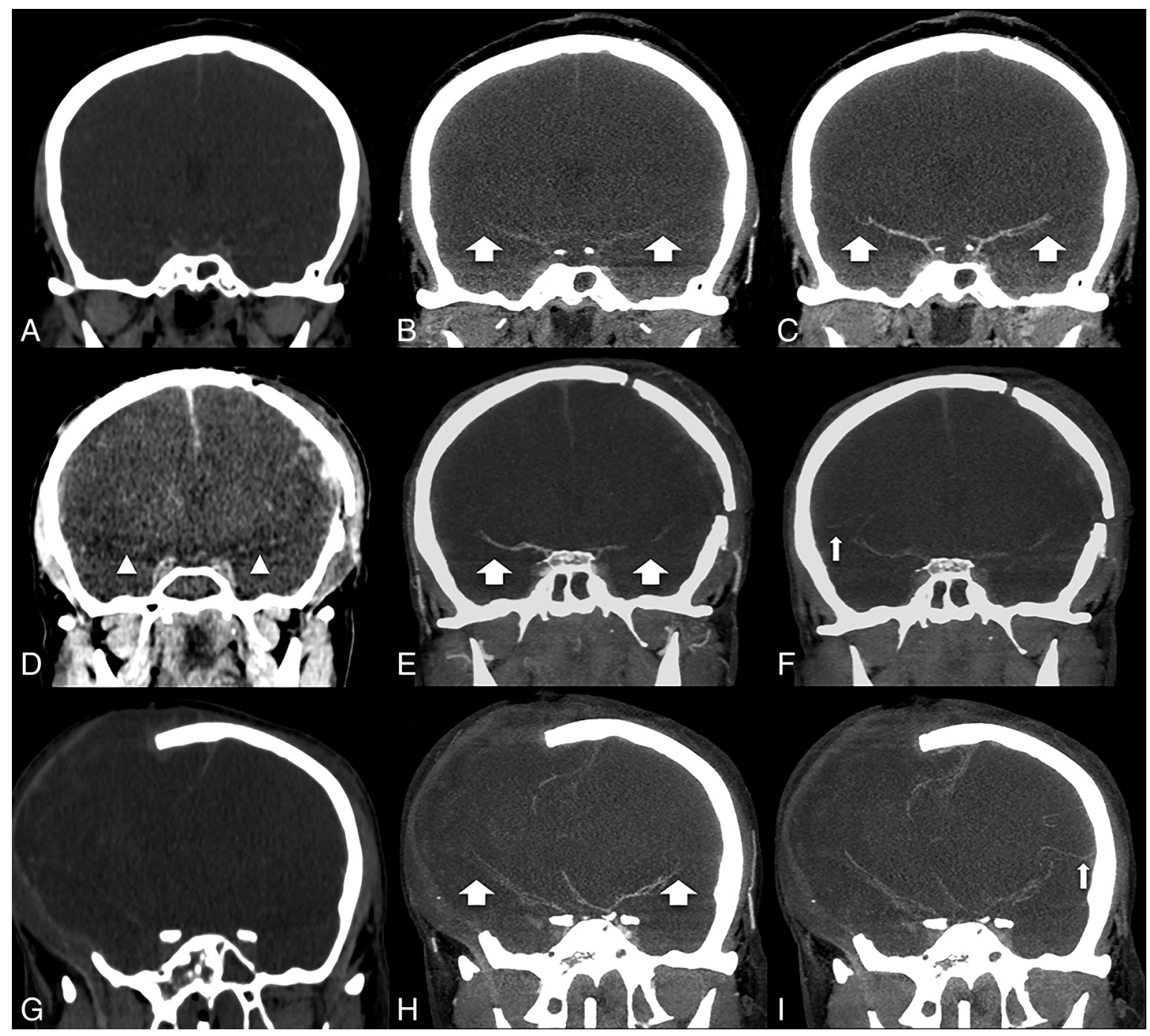

FIG 2. Comparison of $C T$ images without contrast medium $(A, D$, and $G)$ in the arterial $(B, E$, and $H)$ and venous $(C, F$, and $I)$ phases of $C T A$ (MIP reformations in coronal plane) from 3 distinct patients with a brain death clinical diagnosis. In a patient with an intact skull (upper row, $A-C$ ), opacification was observed in the M1-M2 branches (broad arrows) in the arterial $(B)$ and venous $(C)$ phases and was more intense in the late phase. In a patient with craniotomy (middle row, $D-F$ ), one can appreciate the relative hyperattenuation of both $\mathrm{Ml}$ branches mimicking vascular opacification in noncontrast CT (arrowheads in D). Opacification was observed in both M2 branches (broad arrows in the arterial phase, E) and the right $\mathrm{M} 3$ branch in the venous phase (thin arrow in $F$ ), a phenomenon known as stasis filling. In a patient presenting with craniectomy (lower row, $G-l$ ), opacification was observed in both $M 2$ branches (broad arrows in the arterial phase, $H$ ), the right M3 branch, and the left M4 branch in the venous phase (thin arrow in I) due stasis filling, a false-negative case of BD.

with caution, particularly among patients with intracranial hypertension, possibly reducing the specificity of the method. Moreover, the occurrence of deep cerebral venous thrombosis should always be considered to avoid possible false-positive results.

The protocol for BD diagnosis advocated by Frampas et $\mathrm{al}^{12}$ includes image acquisitions of NCCT and biphasic CTA. Alternatively, Sawicki et al ${ }^{18}$ proposed optimizing the CTA technique by performing a single series with an acquisition delay of 40 seconds after the initiation of intravenous ICM infusion. Our study indicated that the maximum progression of the ICM in the intracranial vascular bed occurs during the late acquisition of CTA (venous phase), particularly among patients with concurrent SDs. However, the isolated evaluation of the late phase of CTA potentially limits the differentiation of spontaneously hyperattenuating vascular content, which was often found in our patients with extensive intra- and extracranial edema (pseudo-subarachnoid hemorrhage versus true vascular opacification) because of the SF. This study reinforces the importance of the protocol proposed by Frampas et $\mathrm{al}^{12}$ to avoid mistakes in diagnostic interpretation, thereby enabling comparisons between early and late acquisitions and providing reliable information for the correct evaluation of vascular opacification. The optimization of the interpretation criteria of the images proposed by Frampas et $\mathrm{al}^{12}$ called the modified Frampas criteria, which consist of the absence of opacification of the M4 branches in the arterial phase and ICV in the venous series of the CTA, decreased the number of patients with false-negative findings due to a reduced influence of SF (which is usually present in the arteries during the venous phase), particularly with an SD.

Hemodynamic changes resulting from an SD might represent a confounding factor in the context of $\mathrm{BD},{ }^{10,22}$ thereby necessitating detailed studies to define the limits of the inter- 


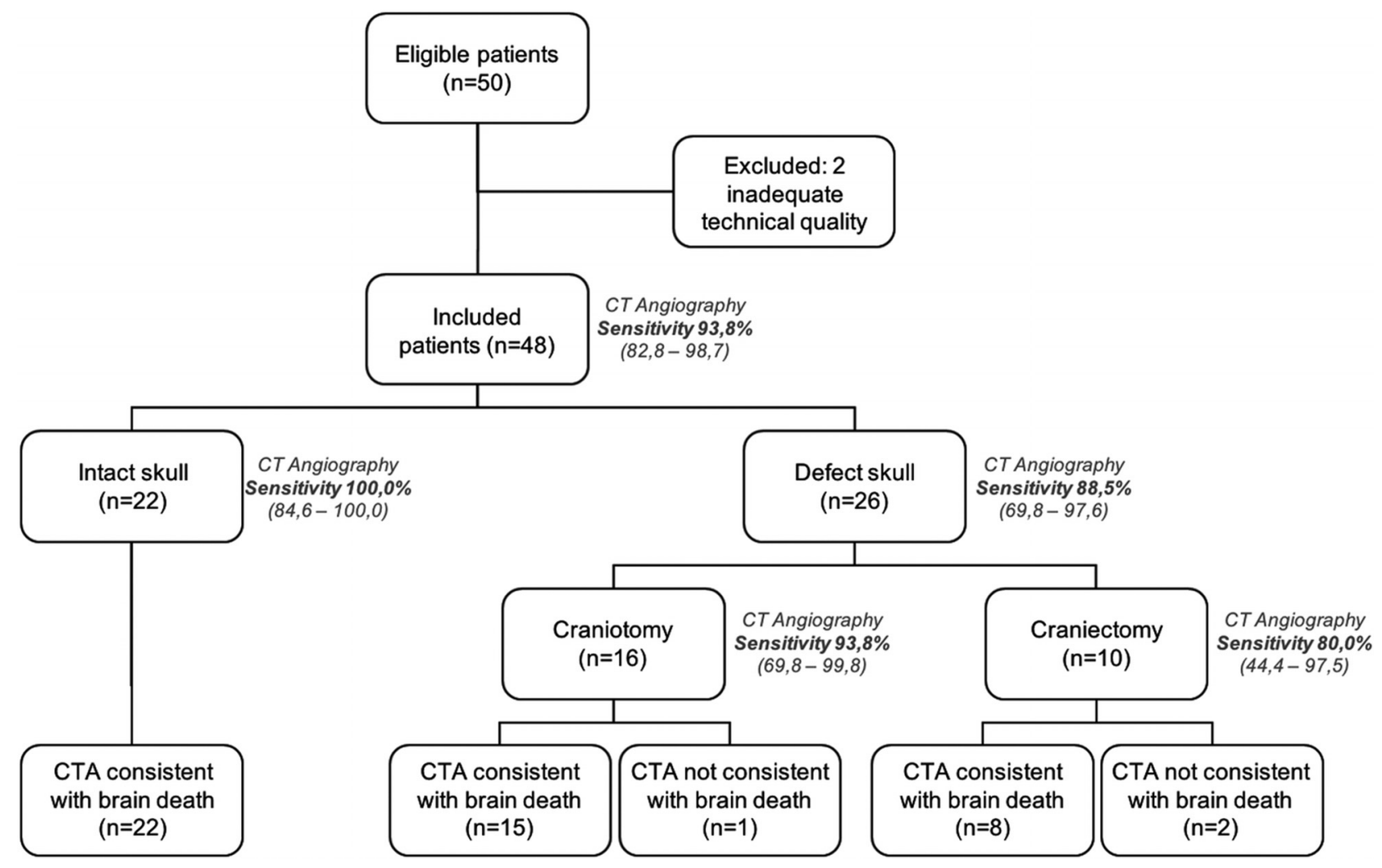

FIG 3. Flow chart of patients with a clinical diagnosis of brain death who underwent the CT angiography protocol proposed by Frampas et al, according to the presence and type of skull defect. Application of the interpretation criteria of the images of the modified Frampas criteria.

pretation for clinical purposes. Two distinct scenarios have been associated with the occurrence of false-negative results during the confirmation of BD in CTA studies: concomitant SD and acute hypoxic-ischemic encephalopathy after cardiorespiratory arrest. ${ }^{12,13,18,22,23,34}$ Our study confirmed that patients with an intact skull had high sensitivity and specificity for confirming BD by CTA, especially when the modified Frampas criteria were used for interpretation of the images, with results similar to those of conventional angiography (the criterion standard). There is a tendency for diagnostic errors to confirm BD by CTA (false-negative results) in patients with DS, particularly craniectomy, when the interpretation criteria of the images of Frampas et $\mathrm{al}^{12}$ were used, probably because of lower intracranial pressure. ${ }^{35-37}$

First described in studies involving conventional angiography, ${ }^{10,26}$ the weak, slow, persistent, and progressive opacification of the intracranial arterial branches by ICM is known as SF. ${ }^{26}$ This finding stems from the progression of intravascular ICM in a high-resistance distal vascular bed without the ability to perfuse the brain parenchyma. ${ }^{13,15,38}$ Recent studies using CTA have indicated a high prevalence of this finding, varying between $45 \%$ and $59 \%$ of all patients with confirmed BD. ${ }^{17,29,38}$ The present results indicated an intermediate rate $(62.2 \%)$ of SF in the total sample when the criteria of Frampas et $\mathrm{al}^{12}$ were used for interpretation of the images. The incorrect diagnosis of BD by CTA was meaningly more common in patients with SF. Despite the small number of cases, this problem tends to occur more frequently in patients with SDs, especially those who have undergone craniectomy, with or without opacification of the venous compartment including the ICV. The interpretation of the images according to modified Frampas criteria decreased the false-negative results and diagnostic mistakes.

Limitations of the present study include intracranial pressure not being measured; thus, establishing quantitative correlations with imaging findings was prevented. CTA to confirm BD also presented some drawbacks, such as the transportation of patients with hemodynamic instability and the difficulty of peripheral venous access in patients with edema.

Strategies to increase the accuracy of CT as a whole in the diagnosis of $\mathrm{BD}$, which might be the object of future studies, include the use of CTP in addition to the standard protocol. CTP is a promising evidentiary method, given the increasing technologic development, the availability of equipment with higher temporal and spatial resolution, and reduced intravenous ICM use. However, some variations in the use of this technique demand the establishment and validation of new standard CTP protocols.

\section{CONCLUSIONS}

This study showed that that the CTA protocol proposed by Frampas et $\mathrm{al}^{12}$ has high sensitivity and reproducibility, as well as perfect specificity and interobserver agreement, for BD confirmation in patients with an intact skull. The optimization of the interpretation of the images according to the modified Frampas criteria increased the sensitivity of the CTA, particularly in patients with SDs. No control case was wrongly diagnosed as BD. These data add to the growing medical literature supporting the use of CTA as a reliable ancillary BD test. A concurrent SD, particularly craniectomy, can decrease the sensitivity of CTA for BD confirmation, mainly due to the SF phenomenon. The evaluation of CTA images 
according to the modified Frampas criteria reduced false-negative findings, especially with craniectomy. Negative results may arise in this clinical scenario, requiring the consideration of other corroborating tests to confirm $\mathrm{BD}$ in patients with SD.

\section{ACKNOWLEDGMENTS}

We extend our appreciation to the Organ Procurement Organization, Santa Casa de Misericordia de São Paulo Hospital, São Paulo, Brazil. The authors thank the institutional publishing support center (Núcleo de Apoio a Publicação) for technical assistance in publication.

\section{REFERENCES}

1. A definition of irreversible coma: report of the Ad Hoc Committee of the Harvard Medical School to examine the definition of brain death. JAMA 1968;205:337-40 CrossRef Medline

2. Wijdicks EF, Varelas PN, Gronseth GS, et al. Evidence-based guideline update: determining brain death in adults-report of the quality standards Subcommittee of the American Academy of Neurology. Neurology 2010;74:1911-18 CrossRef Medline

3. Nakagawa TA, Ashwal S, Mathur M, et al; Society of Critical Care Medicine, Section on Critical Care and Section on Neurology of American Academy of Pediatrics, Child Neurology Society. Clinical report: guidelines for the determination of brain death in infants and children - an update of the 1987 task force recommendations. Pediatrics 2011;128:e720-40 CrossRef Medline

4. Haupt WF, Rudolf J. European brain death codes: a comparison of national guidelines. J Neurol 1999;246:432-37 CrossRef Medline

5. Wijdicks EF. The diagnosis of brain death. $N$ Engl J Med 2001;344: 1215-21 CrossRef Medline

6. Kramer AH. Ancillary testing in brain death. Semin Neurol 2015;35: 125-38 CrossRef Medline

7. Russo H, Bressolle F. Pharmacodynamics and pharmacokinetics of thiopental. Clin Pharmacokinet 1998;35:95-134 CrossRef Medline

8. Marrache F, Megarbane B, Pirnay S, et al. Difficulties in assessing brain death in a case of benzodiazepine poisoning with persistent cerebral blood flow. Hum Exp Toxicol 2004;23:503-05 CrossRef Medline

9. Bradac GB, Simon RS. Angiography in brain death. Neuroradiology 1974;7:25-28 CrossRef Medline

10. Braum M, Ducrocq $X$, Huot JC, et al. Intravenous angiography in brain death: report of $\mathbf{1 4 0}$ patients. Neuroradiology 1997;39:400-05 CrossRef Medline

11. Heran MK, Heran NS, Shemie SD. A review of ancillary tests in evaluating brain death. Can J Neurol Sci 2008;35:409-19 CrossRef Medline

12. Frampas $E$, Videcoq $M$, de Kerviler E, et al. CT angiography for brain death diagnosis. AJNR Am J Neuroradiol 2009;30:1566-70 CrossRef Medline

13. Escudero D, Otero J, Marqués L, et al. Diagnosing brain death by CT perfusion and multislice CT angiography. Neurocrit Care 2009;11: 261-71 CrossRef Medline

14. Berenguer CM, Davis FE, Howington JU. Brain death confirmation: comparison of computed tomographic angiography with nuclear medicine perfusion scan. J Trauma 2010;68:553-59 CrossRef Medline

15. Bohatyrewicz R, Sawicki M, Walecka A, et al. Computed tomographic angiography and perfusion in the diagnosis of brain death. Transplant Proc 2010;42:3941-46 CrossRef Medline

16. Rieke A, Regli B, Mattle HP, et al. Computed tomography angiography (CTA) to prove circulatory arrest for the diagnosis of brain death in the context of organ transplantation. Swiss Med Wkly 2011; 141:w13261 CrossRef Medline

17. Welschehold S, Kerz T, Boor S, et al. Detection of intracranial circulatory arrest in brain death using cranial CT-angiography. Eur J Neurol 2013;20:173-79 CrossRef Medline

18. Sawicki M, Bohatyrewicz R, Safranow K, et al. Computed tomographic angiography criteria in the diagnosis of brain death-com- parison of sensitivity and interobserver reliability of different evaluation scales. Neuroradiology 2014;56:609-20 CrossRef Medline

19. Monro A. Observations on Structure and Functions of the Nervous System. London: Creech and Johnson; 1783

20. Kellie G. Appearance observed in the dissection of two individuals; death from cold and congestion of the brain. Trans Med Chir Soc Edinb 1824;1:84. https://archive.org/details/ b22384315. Accessed December 20, 2018

21. Marshall SA, Kalanuria A, Markandaya M, et al. Management of intracerebral pressure in the neurosciences critical care unit. Neurosurg Clin N Am 2013;24:361-73 CrossRef Medline

22. Alvarez LA, Lipton RB, Hirschfeld A, et al. Brain death determination by angiography in the setting of a skull defect. Arch Neurol 1988;45:225-27 CrossRef Medline

23. Frisardi F, Stefanini M, Natoli S, et al. Decompressive craniectomy may cause diagnostic challenges to assess brain death by computed tomography angiography. Minerva Anestesiol 2014;80: 113-18 Medline

24. Brasil. Conselho Federal de Medicina. Resolução No. 2.173, de 23 de Novembro de 2017. Define os critérios do diagnóstico de morte encefálica. Brasília (DF): CFM; 2017. Disponível em: https:// sistemas.cfm.org.br/normas/visualizar/resolucoes/BR/2017/2173. Accessed December 20, 2018

25. American Association of Physicists in Medicine. The measurement, reporting and management of radiation dose in CT. Report of AAPM task group 23. AAPM report no. 96. https://www.aapm.org/pubs/ reports/RPT_96.pdf. Accessed December 20, 2018

26. Kricheff II, Pinto RS, George AE, et al. Angiographic findings in brain death. Ann N Y Acad Sci 1978;315:168-83 CrossRef Medline

27. Savard M, Turgeon AF, Gariépy JL, et al. Selective 4 vessels angiography in brain death: a retrospective study. Can J Neurol Sci 2010; 37:492-97 CrossRef Medline

28. Taylor T, Dineen RA, Gardiner DC, et al. Computed tomography (CT) angiography for confirmation of the clinical diagnosis of brain death. Cochrane Database Syst Rev 2014;CD009694 CrossRef Medline

29. Dupas B, Gayet-Delacroix M, Villers D, et al. Diagnosis of brain death using two-phase spiral CT. AJNR Am J Neuroradiol 1998;19: 641-47 Medline

30. Leclerc X, Taschner CA, Vidal A, et al. The role of spiral CT for the assessment of the intracranial circulation in suspected brain-death. J Neuroradiol 2006;33:90-95 CrossRef Medline

31. Combes JC, Chomel A, Ricolfi F, et al. Reliability of computed tomographic angiography in the diagnosis of brain death. Transplant Proc 2007;39:16-20 CrossRef Medline

32. Brasil S, Bor-Seng-Shu E, de-Lima-Oliveira M, et al. Role of computed tomography angiography and perfusion tomography in diagnosing brain death: a systematic review. J Neuroradiol 2016;43: 133-40 CrossRef Medline

33. Garrett MP, Williamson RW, Bohl MA, et al. Computed tomography angiography as a confirmatory test for the diagnosis of brain death. J Neurosurg 2018;128:639-44 CrossRef Medline

34. Welschehold S, Boor S, Reuland K, et al. Technical aids in the diagnosis of brain death: a comparison of SEP, AEP, EEG, TCD and CT angiography. Dtsch Arztebl Int 2012;109:624-30 CrossRef Medline

35. Miller K, Eljamel S. Does size and site matter in therapeutic descompressive craniectomy? A laboratory-based experimental study. World Neurosurg 2016;95:441-46 CrossRef Medline

36. Wang YS, Wang Y, Shi XW, et al. Size of bone flap and bone window area may impact the outcome of decompressive craniectomy using standard bone flap. Eur Rev Med Pharmacol Sci 2016;20:3679-82 Medline

37. Bor-seng-Shu E, Figueiredo EG, Amorim RL, et al. Decompressive craniectomy: a meta-analysis of influences on intracranial pressure and cerebral perfusion pressure in the treatment of traumatic brain injury. J Neurosurger 2012;117:589-96 CrossRef Medline

38. Sawicki M, Bohatyrewicz R, Safranow K, et al. Dynamic evaluation of stasis filling phenomenon with computed tomography in diagnosis of brain death. Neuroradiology 2013;55:1061-69 CrossRef Medline 